\title{
The Maximum Opposite Angulation for Mesh Construction
}

\author{
Wen-Jie Cheng ${ }^{\dagger}$, Arzu Gonenc Sorguc ${ }^{\ddagger}$, Junichi Shinoda ${ }^{\dagger}$ \\ and Ichiro HAGIWARA ${ }^{\dagger}$ \\ ${ }^{\dagger}$ Department of Mechanical Science Engineering, Tokyo Institute of Technology, \\ 2-12-1, O-okayama, Meguro-ku, Tokyo 152-8552, Japan \\ $\ddagger$ Department of Architecture, Middle East Technical University, \\ Ankara, Turkey
}

Received November 1, 2002

Revised June 18, 2004

\begin{abstract}
In this paper, a new approach, "the Maximum Opposite Angulation (MOA for short)" algorithm, for the construction of a triangular mesh from a set of unorganized data points is proposed. A topological judgment algorithm is coupled to the MOA algorithm to avoid holes and/or intersections that can be encountered on the reconstructed surface. The MOA algorithm is based on the idea of "the list," which presets a uniformity to the initial data set to fulfill the task of forming "the good mesh architecture" which is defined as the meshes having well balanced interior angles and good aspect ratios. The list, consists of line segments formed by point pairs sorted from the shortest to the longest. The idea of MOA is then to use the shortest line segment as the initial data to start with for reconstruction of a triangular mesh. The vertex point of the triangular geometry is then searched with respect to this line segment to give the good mesh. It is also shown that, the MOA in 3-D which includes the topological judgment in smoothing out the surface of the reconstructed solid model, is superior both in speed and quality, to the existing algorithms.
\end{abstract}

Key words: mesh generation, triangular mesh, topology judgement, scanned data, maximum opposite angulation

\section{Introduction}

In recent years, the production of high quality meshes from a set of unorganized data points has been more and more important. These high quality mesh structures are able to solve many common problems like defining the shape for CAD applications, controlling the surface for CAM applications or allowing computational techniques to be applicable to represent very complicated dynamic or static behaviors. Moreover, these meshes can be applied to develop systems for virtual reality, animation, and computer vision. Another area of application for reconstruction of surfaces is the medical science, the reconstructed models having high precision allow the identification of the pathologies for diagnosis and then treatment through scanned data by CT and MRI. These examples and potential field of applications can be increased exponentially, requiring more complicated and more precise solid models.

In this paper, a new method called as 'The Maximum Opposite Angulation Approach (MOA Approach)' which produces a surface triangular mesh from any given unorganized data points in 3-D space is proposed. It is based on the geomet- 
rical relations of angles and corresponding line-segments forming the sides of the triangles. Besides, since the present method provides meshes with very good aspect ratio and properly balanced internal angles directly, thus, the mesh structures obtained through the use of this method will satisfy the precision and accuracy requirement of FEM employed in the static or dyanamic analysis of any structure. Hence, this newly proposed method can be considered as an efficient technique to provide precise solid models that can be used in many diverse fields where surface reconstruction is an important issue.

\section{Previous Studies}

In this section, before the explanation of the MOA algorithm, a brief outline of the some previous studies is presented. The first study in this field dates back to 1934 , to Delaunay who gave the mathematical foundations of mesh generation. In his paper, 'the good triangular mesh' was described as that with nearly equilateral triangles. Similar discussions were then presented by several researchers in conjunction with the development of the computers.

Studies proposing the planar principles in generating triangular meshes for 2-D applications can be summarized as follows:

Rogers discussed the mathematical background of 'Dirichlet Tessellations' in 1964, then based on this definition, Green-Sibson [12] suggested 'Thiessen Criterion' in the plane. Lawson [17] suggested a criterion, called as 'the Max-Min Angle criterion,' which is basically to divide a quadrilateral into two new triangles in such a way that the diagonal replaces the minimum angle with the possible largest angle. Again Lawson [18] suggested the 'Circle Criterion', based on a circle defined by three points on the circumference, if the fourth point was not inside this circle then these three points were used to construct the triangular mesh. Cline-Renka [8] suggested the 'Opposite Angle Criterion,' which can be explained as connecting a point in the lattice table with a relating triangle to yield a new quadrilateral having internal opposite internal angles larger than 180 degrees. Bowyer suggested 'Circular-ball Standard' for the points lying on the circularity obtained through Thiessen criterion [5]. Sibson [23] showed that the Delaunay triangulation uniquely possessed "Thiessen Criterion" property. He also proved that Lawson's "Circle Test" and "Max-min Angle Property" were actually identical with Delaunay's criterion. In fact, Cline-Renka approach was similarly based on the principle of the Circle Criterion. Actually, in 2-D applications, researchers established the criteria and mathematical principles defining 'the best' in triangular mesh configuration as based on 'the uniformity' of triangle at internal angles i.e. having an equilateral triangular mesh form.

Green-Sibson [12] gave the limit time complexity of Dirichlet tessellations of region division as $O(N \log N)$, where $N$ is the number of data points, the same formula which is considered as the optimum time complexity for 2-D Delaunay class algorithms, can also be observed in the algorithms developed by Lee-Schachter [19] and Cline-Renka [8]. Although these algorithms have the same time complexity, 
their actual computational speeds and thus their computational costs are different. Dwyer [9] suggested the divide-and-conquer algorithm in 1987, which is highly effective. Katajainen-Koppinen [16] suggest to use the quad-tree order algorithm, which has the computational cost near to Dwyer's.

On the ohter hand, there are algorithms adding a point incrementally and changing the decomposition of the region. For such algorithms, it may occur the case that judgement of decomposition of the region is mistaken by numerical errors and the inconsistent topological structure is generated. To overcome such problems, the numerically robust method giving the priority to the topological sturucture, which is called topology-oriented approach, was proposed by Sugihara [24].

In the case of $3-\mathrm{D}$ space, due to complex interventions between the unorganized points and its topology, progress was slow until 1988. Since then, the new approaches in mesh generation in 3-D space appear and almost all of them can be classified into three main categories: surface-based approaches, volume-based approaches and contour tracing approaches based on implicit functions. Choi et al. [7], Bernardini et al. [3], Huan-Menq [15], Bajaj-Xu [2] and Sun et al. [25] are included in the first category. Concerning the second category, volume-based approach, there are works by Boissonnat [4], Amenta et al. [1], Edelsbrunner-Mücke [10] and Guo et al. [13]. The approach based on implicit functions by Hoppe [14] is in the third category.

Among these three categories, surface-based approaches appear to be computationally more effective since a 2-manifold structure is reconstructed incrementally and no intermediate construction of meshes is required. Actually, this idea has roots in the 2-D approaches.

Surface reconstruction was first proposed for objects having relatively simpler topologies. In the ball-pivoting algorithm (BPA) [3], which was developed recently for surface reconstruction from sample points acquired with multiple range scans, the sample points were augmented along the surface normals computed from the range map. Spatial queries were implemented using regular grid of cubic cells with size chosen by the users. But, in this approach, there is the danger of losing the surface original characteristics as shown in Fig. 1, otherwise, in the process of connecting boundary segments, the intersection may appear.

The 3-D Delaunay triangulation principles are utilized in the volume-based
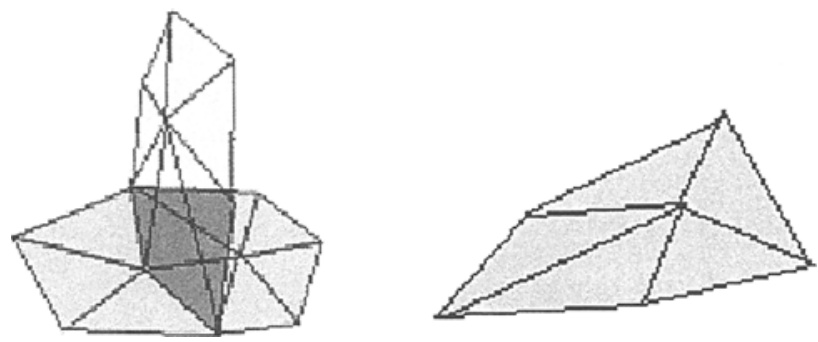

Fig. 1. Problems encountered in connecting the meshes in a different part. 
approaches. In this class of approaches, alpha shapes can be obtained by removing elements having sizes larger than a metric threshold, while crusts can be obtained by Voronoi filtering process. See [10] and [13] for the former, and [1] for the latter. The 3-D mesh generation process consists of the following steps: construction of tetrahedrons, deleting some of the tetrahedrons and finally, obtaining the surface triangular meshes from the tetrahedron solid model. Those algorithms have still some problems related with approximation of the original surfaces and highly computational costs.

In the contour-tracing algorithm [14], the triangular mesh is obtained by contouring the zero set of a signed distance function. In this approach, the size of the region (a data collecting box) where data points are collected, is very important. If the size of the box is not suitable, or, the density of points is low, some features of the object to be constructed cannot be captured. It is also observed that when the unorganized data points do not exhibit uniformity in the spatial distribution, then, the uniform size of the data collecting box is not appropriate anywhere, thus, the reconstructed mesh surface can not interpolate the original input points and can not capture the object geometry precisely. Losing the geometric features of the original surface is an important problem for other implicit function algorithms, as well. In these algorithms, the curvilinear behavior of the implicit functions may alter the original features anywhere on the surface, on the edge or on the corner.

Although there are several approaches in 3-D triangular mesh generation for surface reconstruction, all of them have some problems related with the inherited flaws of the geometrical realization of their algorithms, such as loss of the original characteristics, the distortion in edges and corners, poor quality and intersection, and very high computational costs.

In 3-D applications of mesh generations, the most important issue is to reconstruct the model precisely. Although, there are number of algorithms aiming to provide such models by considering the 'uniformity' of the surface vectors, it is hard to be sure about the best configurations from a set of unorganized data points. It should also be realized that not only the uniformity of the surface vectors, but also the uniformity of the internal angles and the 'size' of the triangles are important.

The uniformity of the mesh architecture is very important and crucial for a precise reconstruction as in the cases of subdivision algorithms, the existing algorithms cannot guarantee the required precision in 3-D space.

In this paper, a method which is called Maximum Opposite Angulation is to be presented, which generates the triangular surface mesh from a set of unorganized data points. The algorithm provided here, aims to get the high precision for the reconstructed models through triangular mesh generation process.

\section{The Maximum Opposite Angulation for Surface Reconstuction}

\subsection{The Maximum Opposite Angulation}

The proposed method 'The Maximum Opposite Angulation (MOA for short)' is based on the well known angle-edge relations of triangular geometries which can 
be stated as the shortest side of a triangle has the minimum opposite angle. In the MOA algorithm, it is aimed to provide geometrical principles of constructing a triangular mesh from an unorganized data point set by choosing candidate points for the vertex of the triangle and the corresponding edge in such a way that the resulting geometry will provide a good aspect ratio and proper balance of internal angles which are the conditions of good meshes as first described by Delaunay. This new method can be explained as follows:

Any initial line segment, yielding a triangle, which is called as 'target line segment,' is formed from a point pair. The third point of the triangle to be constructed is searched in such a way that for the target line segment, the third point results in the corresponding maximum possible opposite angle. This searching method is called as MOA as illustrated in Fig. 2. The third point being the candidate of the vertex for the target line segment is called a maximum opposite point in what follows.

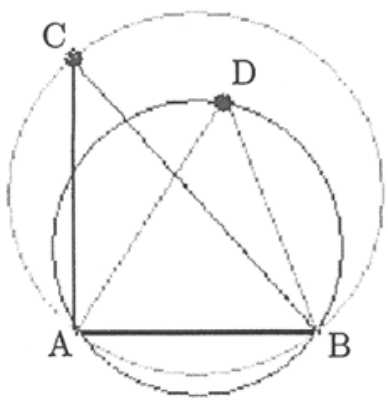

Fig. 2. The Maximum Opposite Angulation MOA.

The basic principle of the MOA algorithm in constructing a triangle is illustrated in Fig. 2. In this figure, $\mathrm{AB}$ is a line-segment obtained by connecting a point pair, and $C$ and $D$ are the points searched for obtaining the vertex of the triangle in order to yield the maximum angle for the line segment of interest, namely $A B$. When these points are connected with $A B$, then $A C, B C, A D$ and $B D$ are formed, but among all these connections, as shown in Fig. 2, there exists one possible choice, which is the angle $\angle \mathrm{ADB}$ as the maximum opposite angle, enabling the construction of triangle $\mathrm{ABD}$.

In 2-D case, from the target line segment, the searching of the point resulting in maximum opposite angle can be considered as searching the smallest circle passing through the searched possible vertex point and the endpoints of the target line segment. In 3-D case, instead of the smallest circle, smallest cardioid-like shape is to be searched. The target point providing the maximum angle lies on the surface of cardioid-like shape.

\subsection{The concept of 'good mesh' in 3-D}

In 3-D mesh generation, the most important issue is to reconstruct the model precisely. However, it is very difficult to provide required precision and smooth 
architecture by preserving the correct topology due to the complicated nature of 3-D problems. Although, there are a number of algorithms aiming to provide such models by considering the 'uniformity,' based on the surface normal vectors, it is hard to be sure about the good configurations from a set of unorganized data points. It should also be realized that, not only the uniformity base on the surface normal vectors, but also the uniformity of the internal angles and the "size" of the triangles are important.

Though the uniformity is very important and crucial for a precise reconstruction, the existing algorithms cannot guarantee the required precision in 3-D space. The effort is to improve this situation and the method proposed in this paper, appears to be more precise with the geometrical principles discussed above in order to provide good mesh architecture. The advantages of the MOA algorithm in 3-D surface reconstruction are to be discussed in the next section and some examples are to be given.

\subsection{Problems encountered in 3-D space and principles of mesh gener- ation by MOA}

If using the MOA algorithm without other judgments, MOA also has some problems related with the intersection phenomenon in 3-D surface reconstruction. The MOA algorithm, which sets a pre-set uniformity to point data sets and combines the shortest line segments with points yielding the maximum angle aiming 'the good mesh,' may not guarantee the completeness of the topology but it identifies and prevents certain type of intersections adequately. The type of intersections that can be observed in 3-D triangular meshes is classified into three groups as shown in Fig. 3. Type 1: one triangle passes through the interior region of other triangle; Type 2: there exists a submesh homeomorphic to the Möbius band in the original mesh; Type 3: one of the edges in the mesh is shared by more than 2 triangles, Type 1, 2 and 3 correspond to Fig. 3 (1), (2) and (3), respectively.

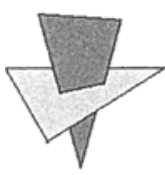

(1)

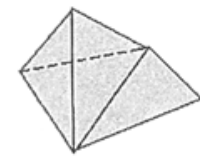

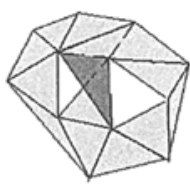

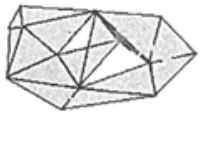

(2)
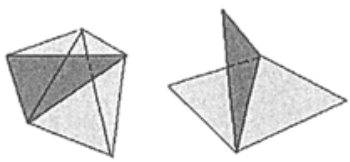

(3)

Fig. 3. The most possible intersections observed in 3-D space.

As for the intersection of type (1), the geometrical construction employed in the MOA algorithm prevents the formation of this type of intersection. In fact, 


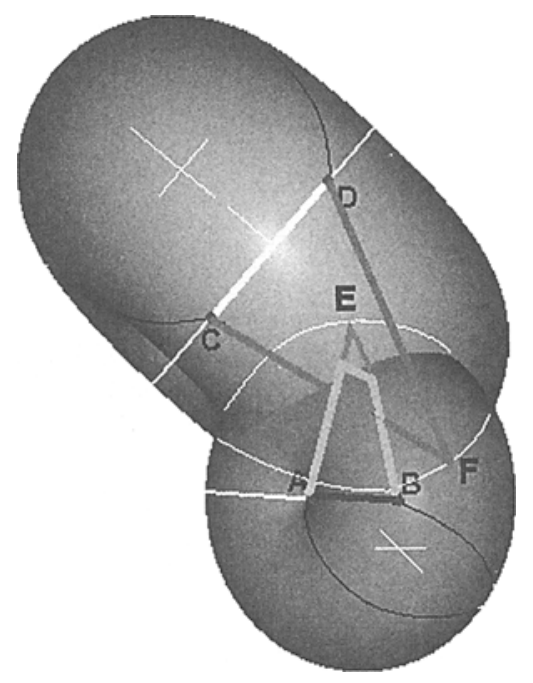

Fig. 4. Avoidance of intersection Type 1 by MOA algorithm in 3-D space.

this is shown as follows. Let $\mathrm{AB}$ and $\mathrm{CD}$ be two target segments and, $\mathrm{E}$ and $\mathrm{F}$ be related maximum opposite points, respectively. See Fig. 4. This construction makes impossible to have points in this region, so intersection phenomenon of Type 1 is avoided.

Let us define the folded structure. For a pair of triangles sharing one edge or one vertex, if the neighboring structure can be extended to that of Type 3 , we call such structure the folded structure. The definition is not rigorous but the reader can easily understand it from Fig. 6, which shows the typical examples of folded structure. In order to identify the possibility of occurrence of such structure, it is necessary to discuss the geometric construction employed in the proposed algorithm.

The intersection can be considered as the result that inner segment of the triangular mesh is connected with the different part of the same mesh. If we can find beforehand where folded structures may occur, intersections can be prevented in the initial stage.

The MOA algorithm in 3-D space, due to its geometrical construction mechanism may result in two different combinations of triangle pairs. which may lead to the intersection. The first possible combination of triangles illustrated in Fig. 5 (1) occurs when two triangles share the same target line segment. This is actually the most common type. The second possible combination of triangles illustrated in Fig. 5 (2) occurs when the maximum opposite point is the endpoint of the other. When such relations occur in some region, region may not be connected properly with other neighboring regions, thus folded structure may be observed as shown in Fig. 6.

Although there are some possibilities encountering the intersection problem in the MOA algorithm, the topologic analysis and the generation process separate from it will help us avoid the intersection and also construct the superior mesh. In this 


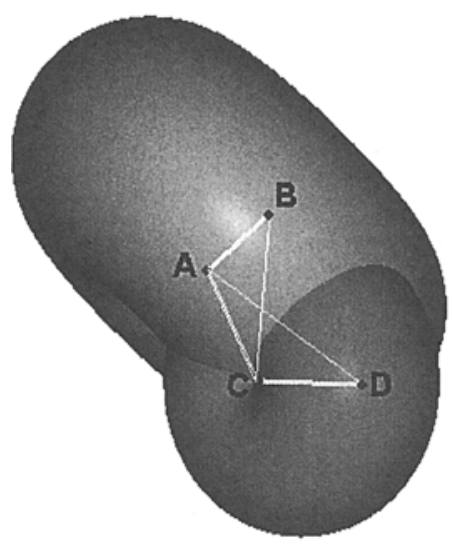

(1)

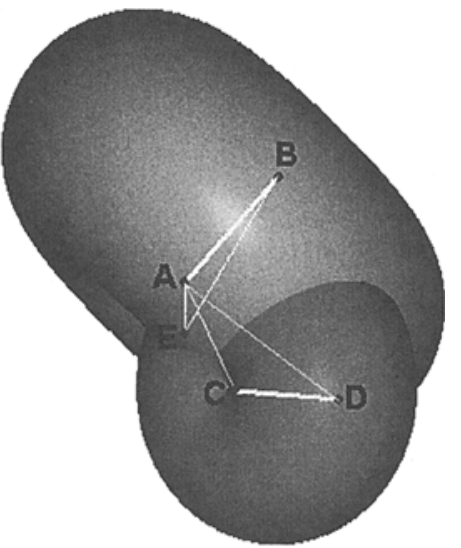

(2)

Fig. 5. The relation of connection of two triangles due to MOA algorithm which may lead to the intersection.

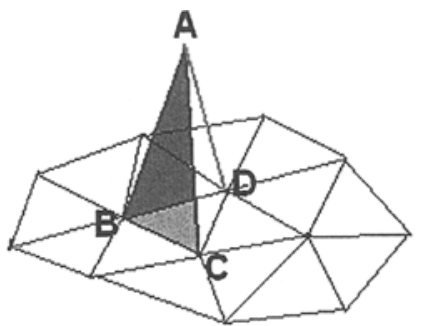

(1)

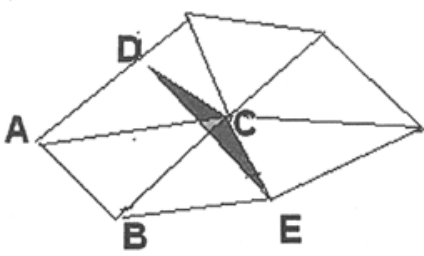

(2)

Fig. 6. Folded structures in 3-D space due to the MOA algorithm without any judgment.

paper, an additional algorithm related with the topology judgment is introduced to the MOA algorithm. This new approach is to be discussed in the next section.

\subsection{The topologic judgment and MOAA in 3-D space}

The surface reconstruction is a task that should be accomplished according to needs and purposes. Therefore, the algorithm to be used is a compromise of all the requirements like precision, computational cost, smoothness and so on. The same argument is also valid for the mesh optimization and several different approaches can be employed simultaneously to obtain "the best" mesh architecture.

Most of the surface reconstruction algorithms in 3-D space encounter the problem of intersection. As it is discussed previously, the intersection phenomenon observed in the MOA algorithm arises from having line segments shared by more than two triangles. In this section, a new approach using topological judgment is to be introduced to improve the precision of the MOA algorithm in 3-D surface reconstruction. 
The folded structure occurring on the reconstructed surface can be identified by checking the topological characteristics of the surface. More precisely, to do this, the relation between the target triangle and its surrounding data points is checked. This type of evaluation can only be realized for the "finished" triangles. Here a finished triangle means the triangle just obtained by MOA from the target line segment. The procedure is as follows:

The reference plane is determined by three vertices of the finished triangle. Let $\mathrm{D}$ be a center of this triangle and, for any point $\mathrm{P}$, let $\mathrm{I}$ be its projection to the reference plane. Now for any two points $A$ and $B$ we denote by $|A B|$ the Euclidean distance between these points. Then for a finished triangle we can define a topology judgment function $T$ of the form

$$
T=\left(\frac{|\mathrm{PD}|}{b}\right)^{2} \times\left(\frac{|\mathrm{PI}|}{|\mathrm{DI}|}\right)^{c}
$$

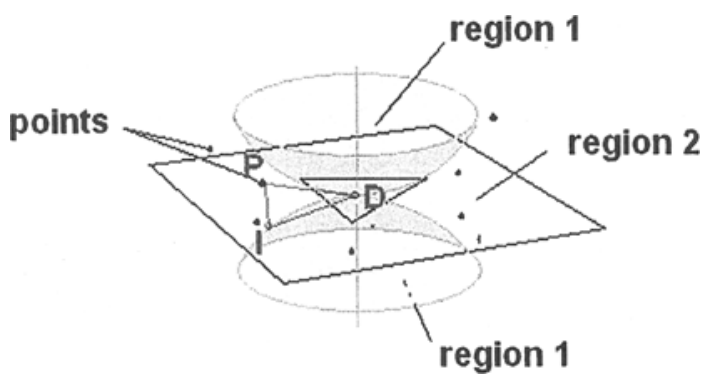

Fig. 7. The identification of folded surfaces through the topology judgment based on a triangle.

where $b$ is a parameter depending on this triangle and $c$ is a positive constant. In case $b$ is the average length of three edges of the triangle and $c=1,\{\mathrm{P} \mid T<a\}$ is the region bounded by a curved surface as shown by region 2 in Fig. 7 . Moreover, the smaller the finished triangle is, the narrower the region $\{\mathrm{P} \mid T<a\}$ is. So if $a$ is small and all the neighboring points are included in that region, this means that neighboring points of $\mathrm{D}$ are distributed as if they were on some smooth surface and hence a folded structure may not occur around point $D$.

Since the fact similar to the above example holds for general $T$, it is easily seen that this function $T$ can identify the folded structures. The same approach can also be used to identify the points on the corner or on the edge by choosing point $D$ as one of the vertices of the triangle instead of its center.

Another topology judgment of folded structures is to control the angles arising from pairs of triangles. As noted earlier, the pair of triangles shares either a point or an edge, if they have a common part. When the triangle pair shares one point, say $\mathrm{P}$, as shown in Fig. 8 (1), let $\alpha$ be the angle of two vectors $\overrightarrow{\mathrm{PM}}$ and $\overrightarrow{\mathrm{PN}}$, where $\mathrm{M}$ and $\mathrm{N}$ are the center of the triangles, respectively. If $\alpha$ is small enough, the 


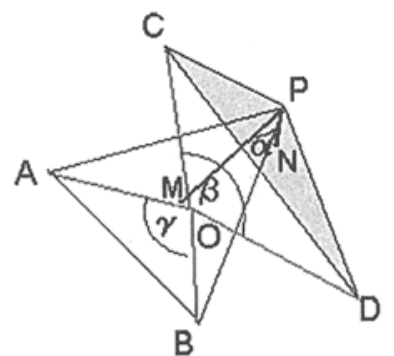

(1)

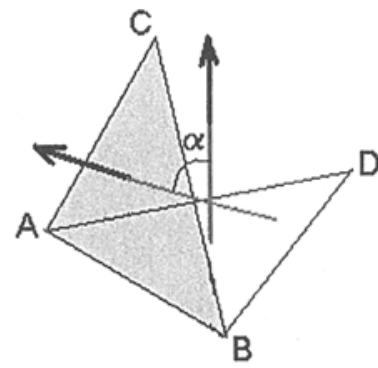

(2)

Fig. 8. The judgment of folded structures by angles arising from a pair of triangles.

pair of triangles are considered to be duplicate as shown in Fig. 6 (2), hence they may proceed to be a folded structure. In case that the aspect ratio of one of the triangles is very bad, $\alpha$ may also be small even if there is no folded structure. So the supplementary judgment is necessary in that case. Connecting the center $O$ of the four points $A, B, C$ and $D$, with two vertices, different from point $P$, of each triangle, we get two angles $\beta$ and $\gamma$, see Fig. 8 (1). In addition, if both of these angles are large, the triangle pair probably produces the folded structure.

When the triangle pair shares one edge, say $\mathrm{AB}$, let $\alpha$ be the angle of two vectors $\overrightarrow{\mathrm{AB}} \times \overrightarrow{\mathrm{BC}}$ and $\overrightarrow{\mathrm{AB}} \times \overrightarrow{\mathrm{BD}}$, where $C$ and $D$ are vertices of the triangles different from $\mathrm{A}$ and $\mathrm{B}$ as shown in Fig. 8 (2). If $\alpha$ is small, then this is a topological indication of existence of the folded structure. The simple geometrical realizations discussed above as topology judgment of existence of folded structures due to the MOA algorithm, improve the efficiency of the proposed algorithm with higher precision to the objective models.

Thus, in implementing the MOA algorithm in the 3-D surface reconstruction, the topological characteristics are also checked simultaneously with the generation process to avoid the defects on the surface to be reconstructed. This improvement is to be illustrated by examples in the next section.

When these folded structures are identified during the mesh generation process, the MOA algorithm clears out those data points yielding such structures. However, this elimination itself may cause some problems related with loss of features of the original surface, and some "holes" may appear on the surface at the end as shown in Fig. 9.

These holes are also treated by the "hole module" of the improved MOA algorithm, which is basically another topological judgment routine. This hole module can be considered as a post-improvement tool for the reconstructed surface. Basically, in this algorithm, the boundaries of each hole formed by the segments, each of which belongs to only one triangle, of the triangular mesh are identified. More precisely, if the hole configuration is as shown in Fig. 9 (1), the hole is recognized by the line segments, each of which belongs to only one triangle, connected consecu- 

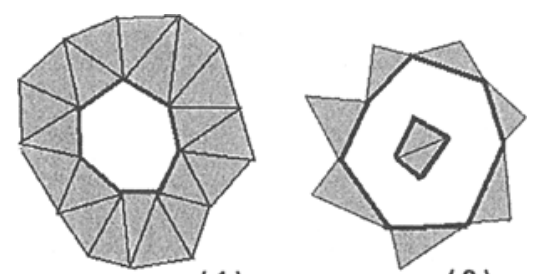

(1)

(2)

Fig. 9. Possible types of holes on the mesh.

tively along a chosen loop direction. In case that the hole configuration is as shown in Fig. 9 (2), the procedure is very similar to the previously described one, but, inner and outer loops are recognized, respectively, and the line segments forming each boundary of the hole are connected properly. Experimentally, the second case is very rare.

\subsection{MOA algorithms in 3-D mesh reconstruction}

In this section, the MOA algorithm for 3-D space is to be outlined. MOA for 3-D space can be considered as a two step algorithm, each of which is to aim to control the possible problems explained in the previous section. It is also necessary to explain "the priority gradation" to make clear the implementation of MOA in 3-D space. It can be considered as a topological judgment technique, to guarantee the quality of meshes (like the balance of interior angles and thus the good aspect ratio, and the smoothness of the whole structures, etc.). In the following algorithms of MOA in 3-D space, this technique is employed implicitly throughout the mesh construction process, to avoid the occurrence of intersections and holes on the surface and moreover to provide the "good meshes."

MOA for 3-D mesh generation does not adopt the method of expanding the boundary of the existing mesh gradually since such kind of method may lose the geometric features.

\subsubsection{Algorithm 1 of MOA for search for the Maximum Opposite Angle}

3-D MOA algorithm is described in the following two parts. The first one is the initial mesh construction. If the set of initial points are suitably distributed, mesh construction process is completed in this step. From the consideration in 3.4, the algorithm is summarized as follows:

1. All the initial data points are ordered in the space by octree algorithm.

2. For any point, one of the nearest neighbors is selected among relevant tree structure and they are connected. All such line segments are listed in the table according to their length. The priority of shorter line segment is higher than that of longer one.

3. A sphere of radius $R$ centered at the mid-point of the target line segment is determined. Among relevant points in the tree, the maximum opposite point is selected for constructing one reference plane. Based on this plane, the points inside the sphere are evaluated by the topology judgment function $T$. 
And for each neighboring triangle sharing one point (resp. one edge), angles $\alpha, \beta$ and $\gamma$ (resp. angle $\alpha$ ) defined in 3.4 are (resp. is) determined.

4. If all values of $T$ are small enough, all $\alpha$ are small and all $\beta$ and $\gamma$ are large for relating points or triangles, this triangle is determined and new constructed segments are included in the segment list according to their length. Otherwise, this triangle is not included in the list and go back to Step 3 for the next segment.

5. By the plane vertical to the reference plane and passing through the base segment, the set of the points inside the sphere is divided into two groups.

6. Then from the point group not including the point obtained in Step 3, the maximum opposite point selected to the same base segment for the construction of a new triangle if it exists to the base segment. If the rationality of the new triangle is confirmed by the topology judgment same as in Step 4, newly constructed segments are set into the segment list according to their length and change the target segment to the next one in the list.

7. Repeat Steps 3-6 until all the segments in the list have been checked.

\subsubsection{Algorithm 2 of MOA for loops}

The Algorithm 1 is not enough to complete the surface reconstruction because the avoidance of intersections may result in some holes on the surface as noted before. In order to remove these holes, the following Algorithm 2, "Loop algorithm" is developed. The octree algorithm is also used for reducing the number of irrelevant triangles and segments; we search triangles and segments that belong to the relevant region determined by the tree structure.

1. If, in the region surrounded by the loop, there exists a line segment having one common point with other boundary line segments, it can also be regarded as a loop by counting this segment twice and has the priority of mesh generation in loop segments. If there exists a line segment without having any common point with other boundary line segments, its endpoints have the priority to be selected.

2. All "loops" of holes are recognized by the line segments connected consecutively along a chosen direction. The segments are stored in the "list." If the hole has the form shown in Fig. 9 (2), then, the segments forming the inner and the outer boundary of the hole should be stored in two different lists. Actually, this step is the same for all types of holes; the difference is only the number of lists.

3. For a fixed loop, based on the shortest segment of this loop, using the MOA algorithm, the new triangles are constructed only by the points in the list of the loop of interest.

4. The creation of a new triangle gives new holes, each of which is to be filled. This procedure is shown in Fig. 10. If the loop is the first type, the resulting loop has two possibilities as shown in Fig. 10 (1) and (2). If the loop is the second type, the resulting loop is considered as a loop of first type as shown in Fig. 10 (3), hence the two lists are combined into one. But the selected point is 


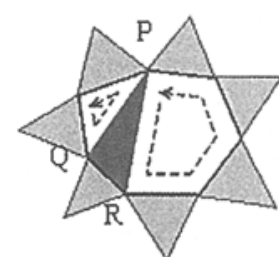

(1)

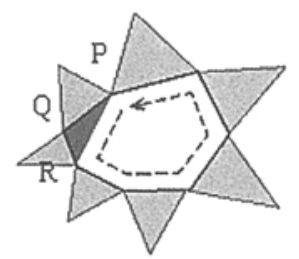

(2)

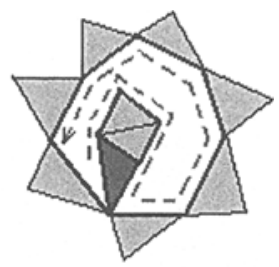

(3)

Fig. 10. Triangulation inside the loop.

discarded if the resulting triangle is not compatible with the original structure. This step is repeated many times until the hole is completely meshed.

5. Repeat Steps 3-4 until all loops are checked.

\section{Case Studies}

In this section, MOA algorithm in 3-D space is used to reconstruct the one of the statues found in the Easter Island as shown in Fig. 11.
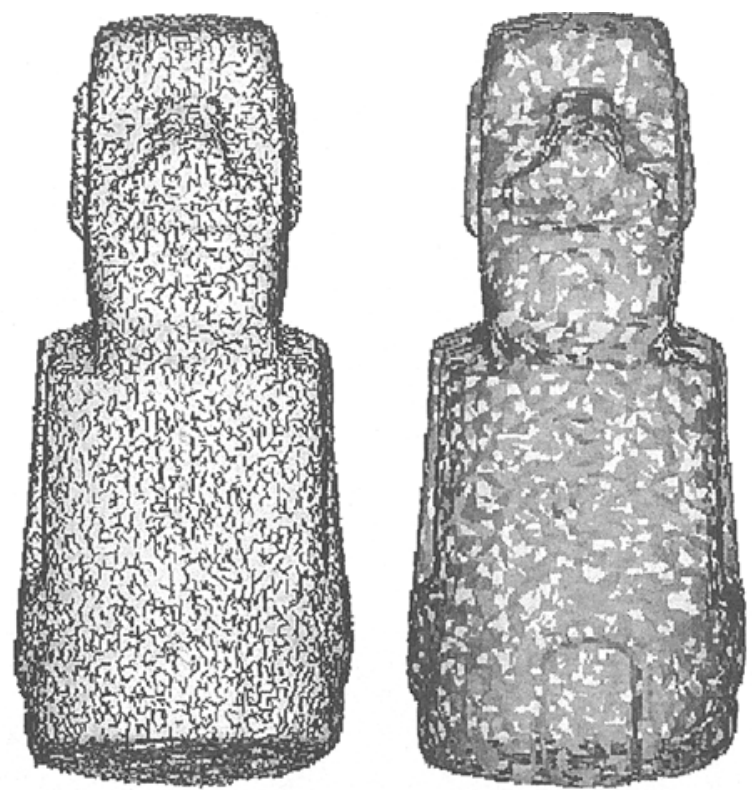

Left hand side, the formation of initial linesegments

Fig. 11. The reconstruction of the Easter island statue through 3-D algorithm 1 of MOA.

The reconstructed model is obtained through a set of unorganized data points. The number of the data points employed in the reconstruction is 10002 , the number of line segments is 30012 and the number of triangles is 20006 . During the mesh generation process, the topology judgment to identify and remove intersections, are combined with the original MOA algorithm. The resulting solid model, illustrated 
in Fig. 12 contains many holes, as expected, on the surface. The reasons of these holes are already discussed in the previous section.

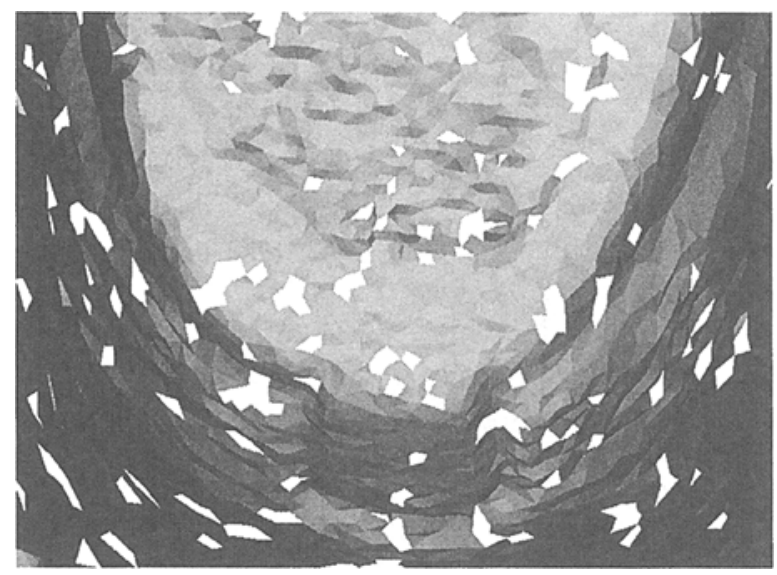

Fig. 12. Close look to the holes on the reconstructed surface.

In order to get a complete and precise reconstructed model as it is explained, these holes should be removed from the reconstructed surface. This is achieved by using the hole module of the MOA in 3-D space and the resulting model is shown in Fig. 13.
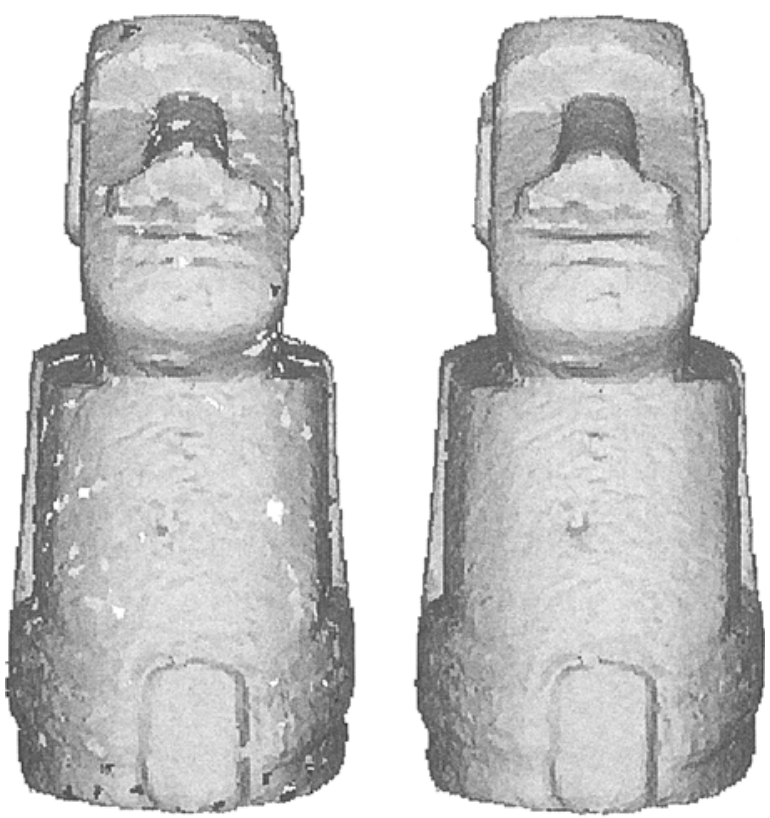

Points: 10002, Segments: 30000, Triangles: 20000

Fig. 13. The progress in the reconstruction and the final from of the solid model. 

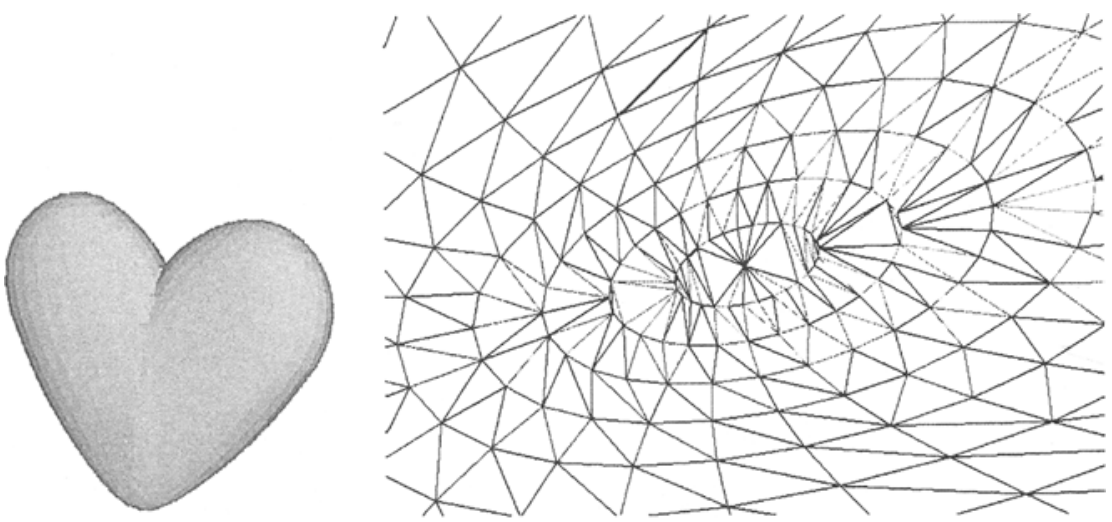

Fig. 14. Abstract heart reconstructed by MOA.
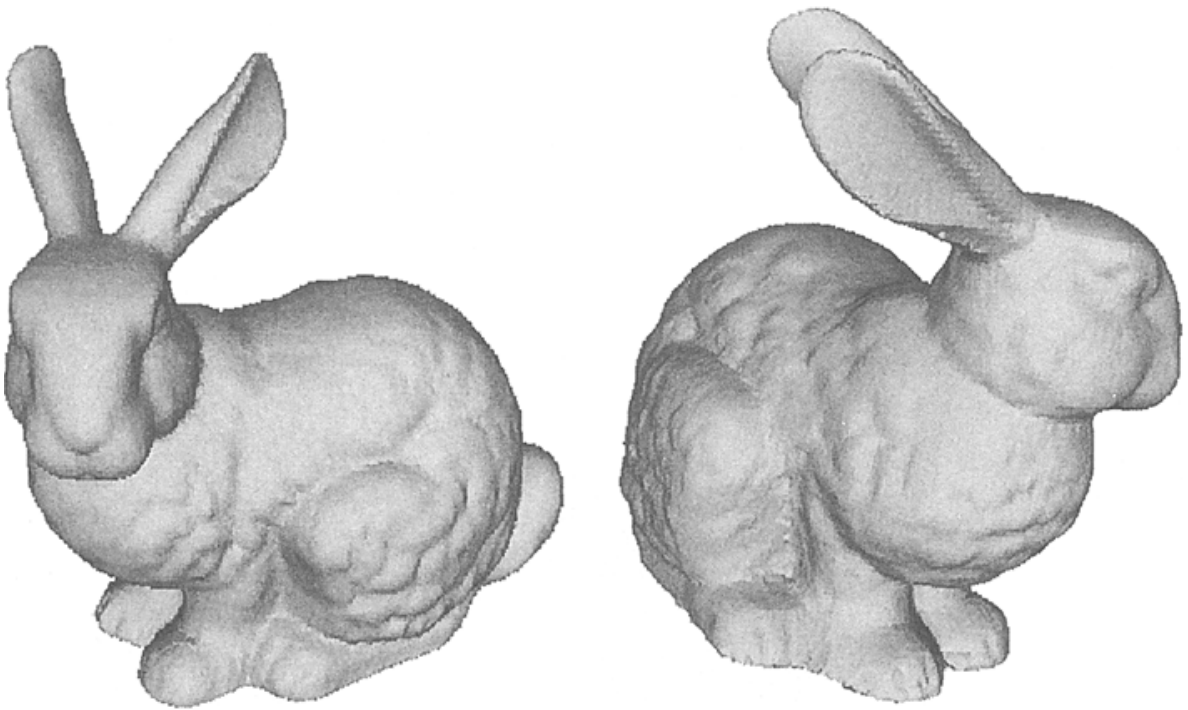

Points: 35947, Segments: 107835, Triangles: 71890, Speed: P4-1.6G/32.12 minutes

Fig. 15. Bunny reconstructed by MOA.

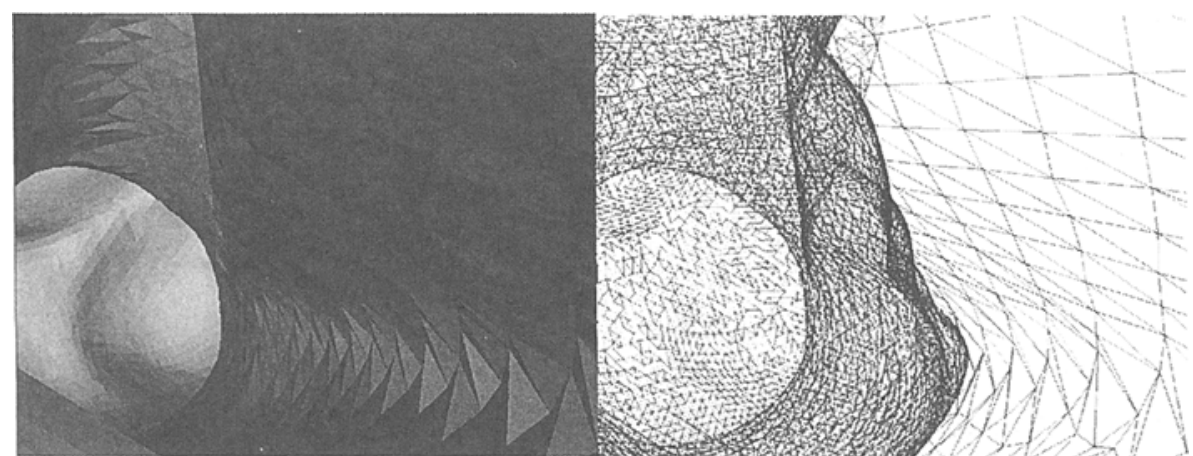

Fig. 16. A close look to the mesh architecture inside of the reconstructed bunny's ear. 

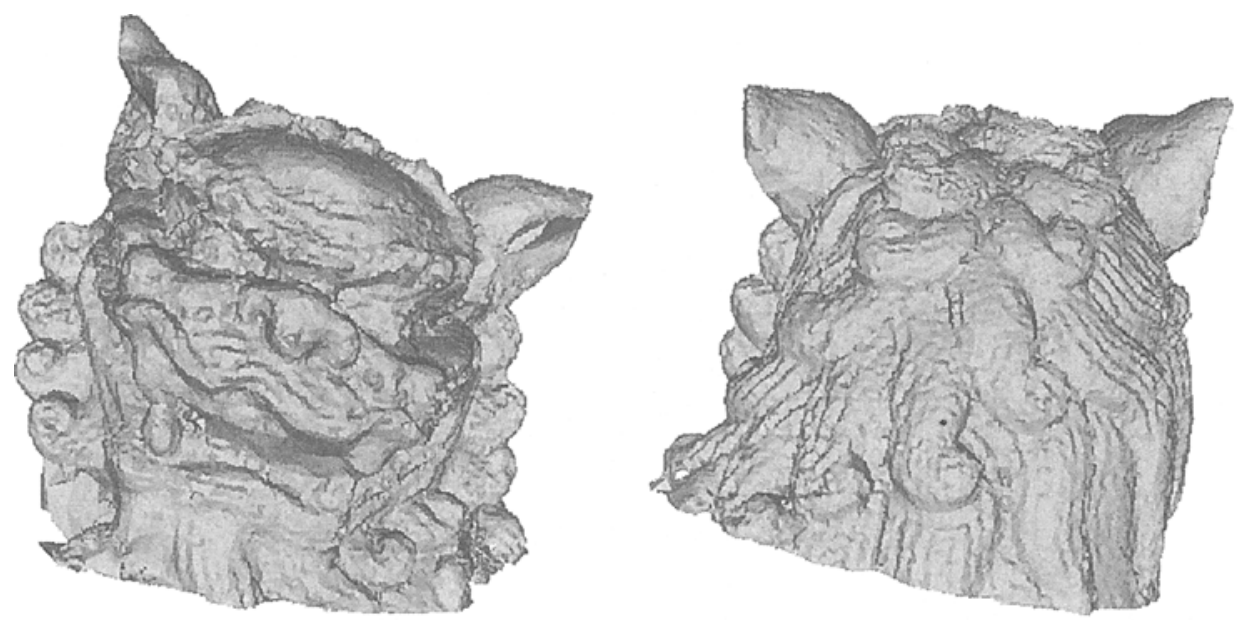

Points: 24930, Segments: 74784, Triangles: 49856, Speed: P4-1.6G/16 minutes

Fig. 17. Chinese lion-dog reconstructed by MOA.

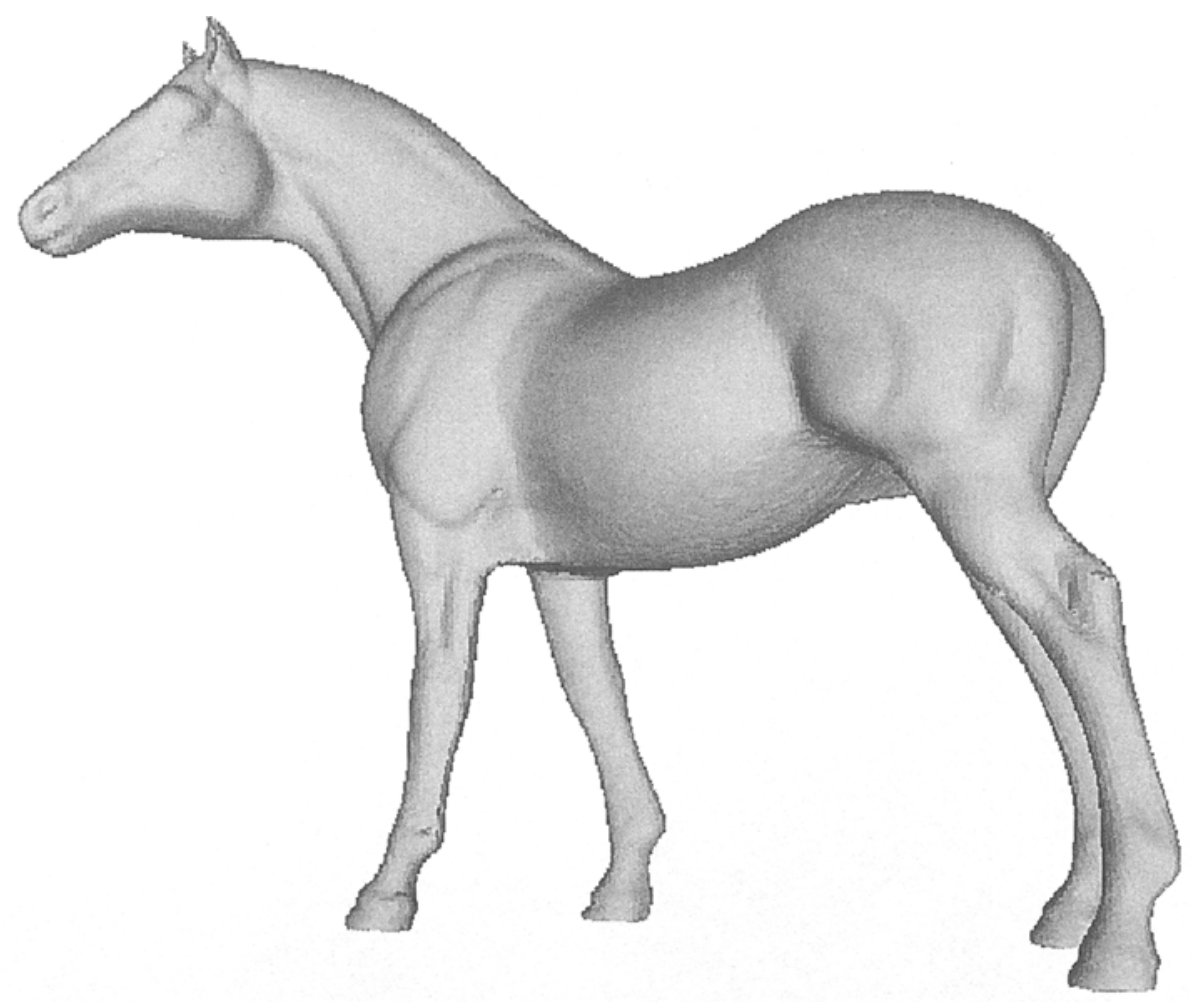

Points: 48485, Segments: 145499, Triangles: 96966, Speed: P4-1.6G/39.33 minutes

Fig. 18. Horse reconstructed by MOA. 

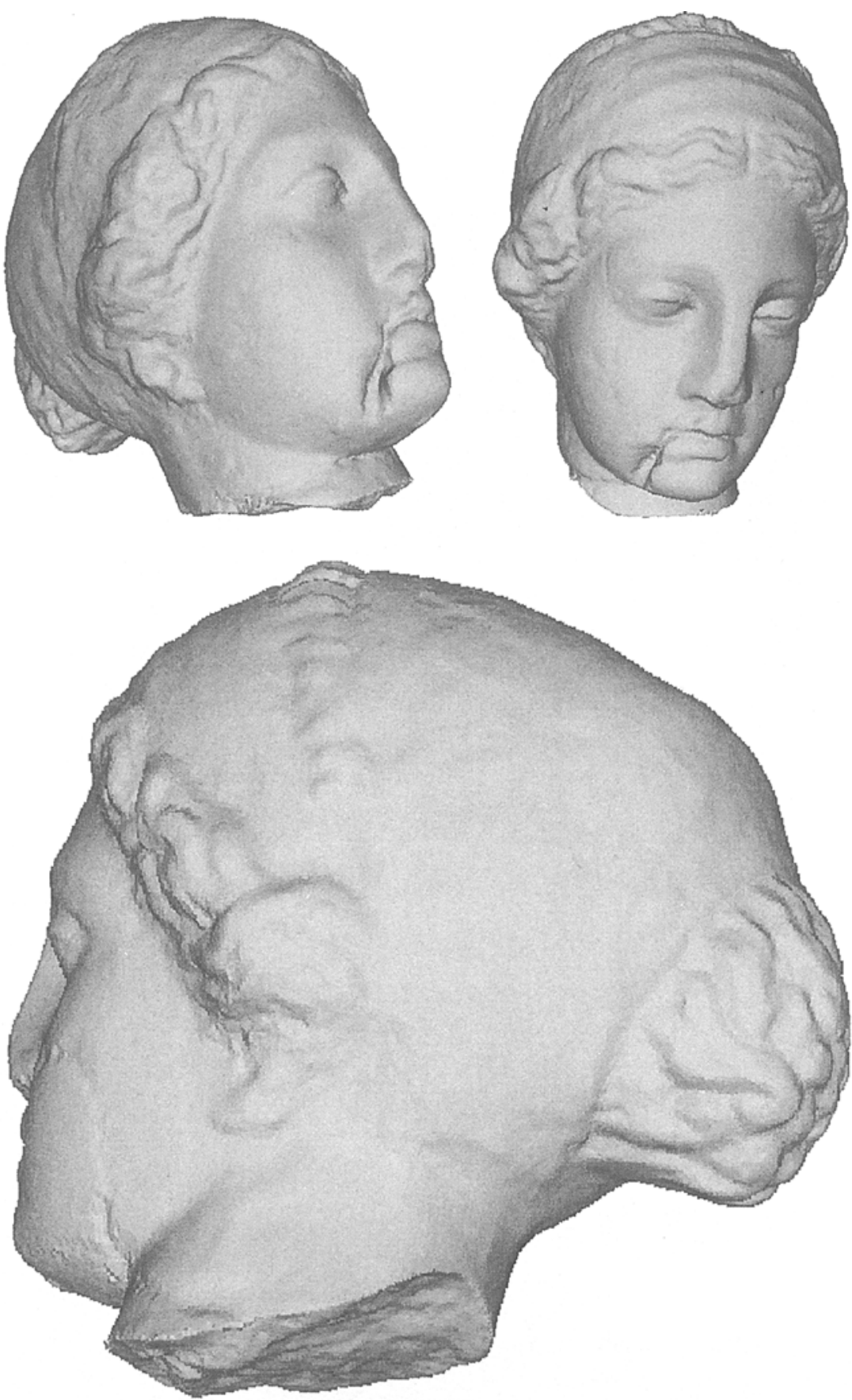

Points: 67180, Segments: 201534, Triangles: 134356, Speed: P4-1.6G/59 minutes

Fig. 19. Venus reconstructed by MOA. 


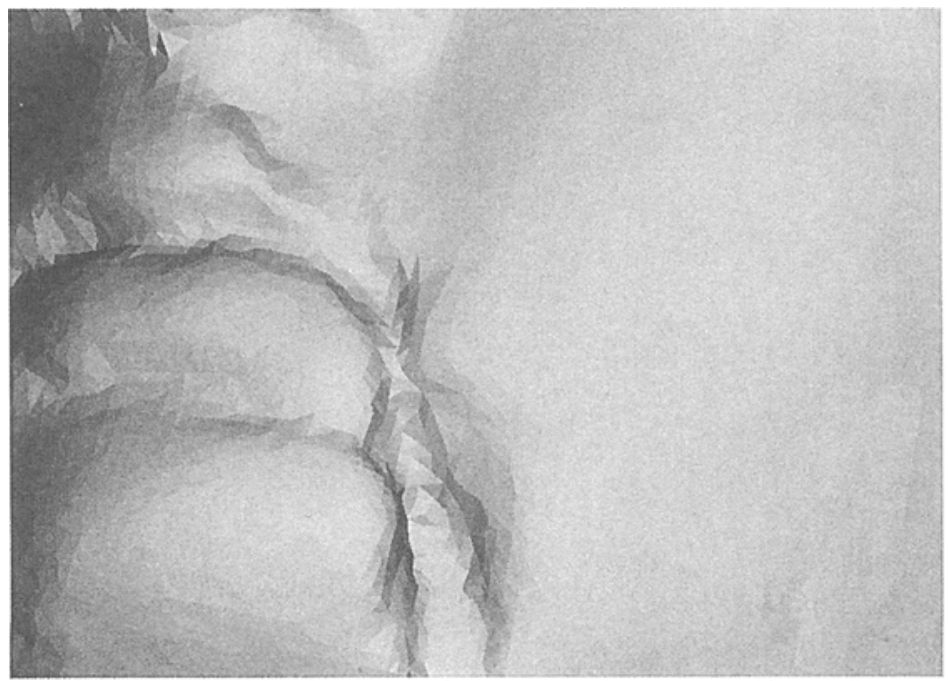

(1) The original surface of Venus model

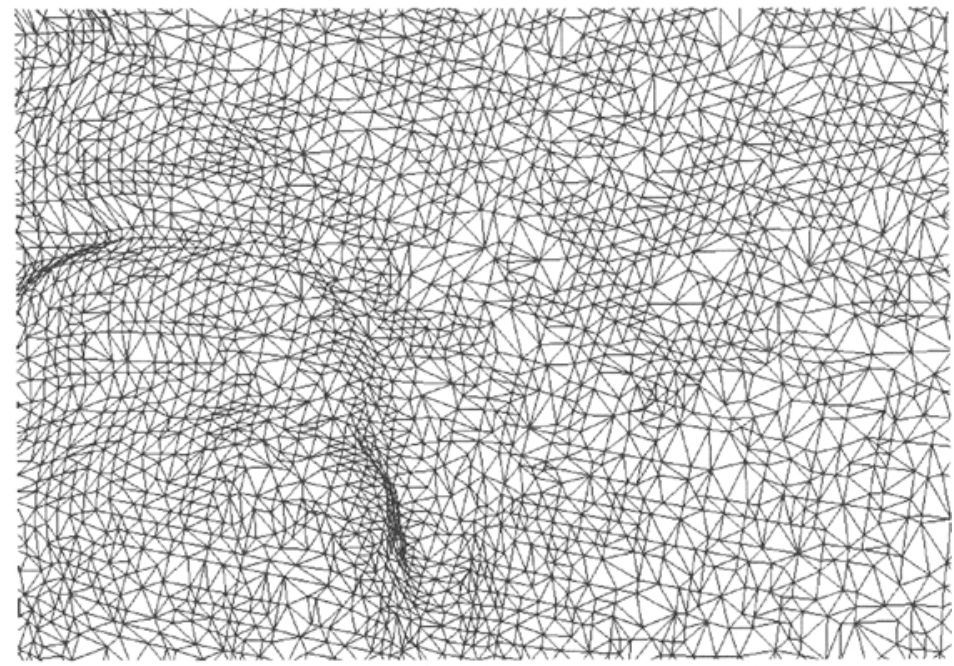

(2) The original surface mesh structure

Fig. 20. The part of the mouth of the original Venus model. 


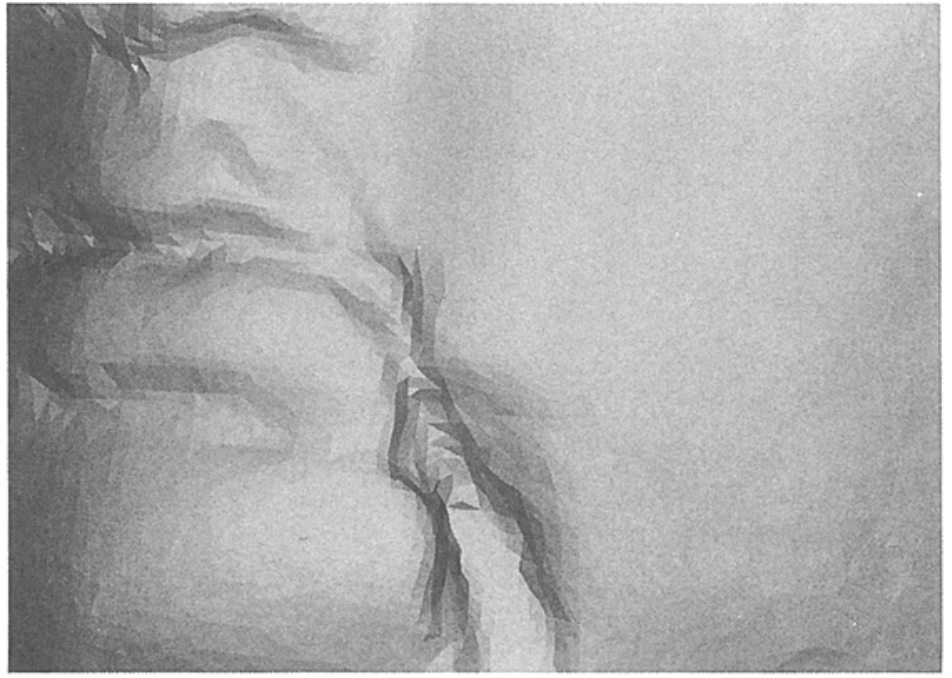

(1) The surface reconstructed by MOA algorithm for 3-D space

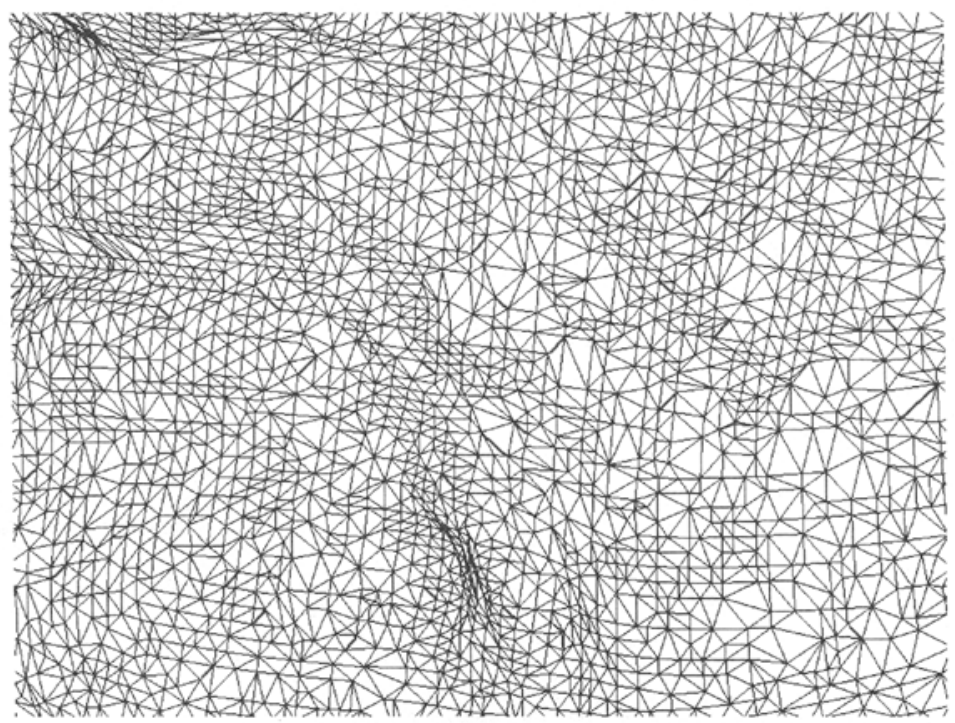

(2) The mesh structure by MOA

Fig. 21. The part of the mouth of Venus by MOA. 

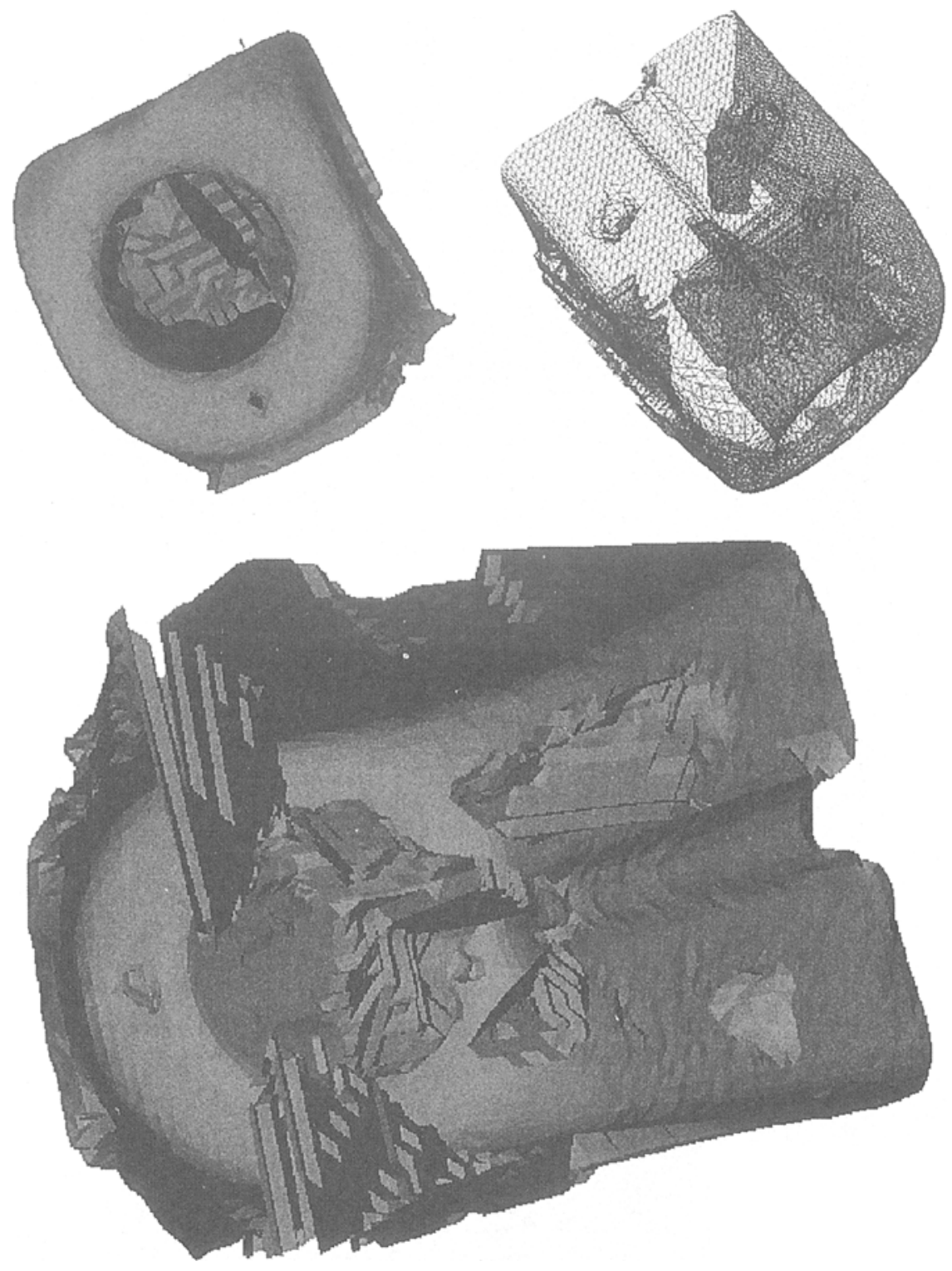

Fig. 22. The results by Hoppe's approach for surface reconstruction. 

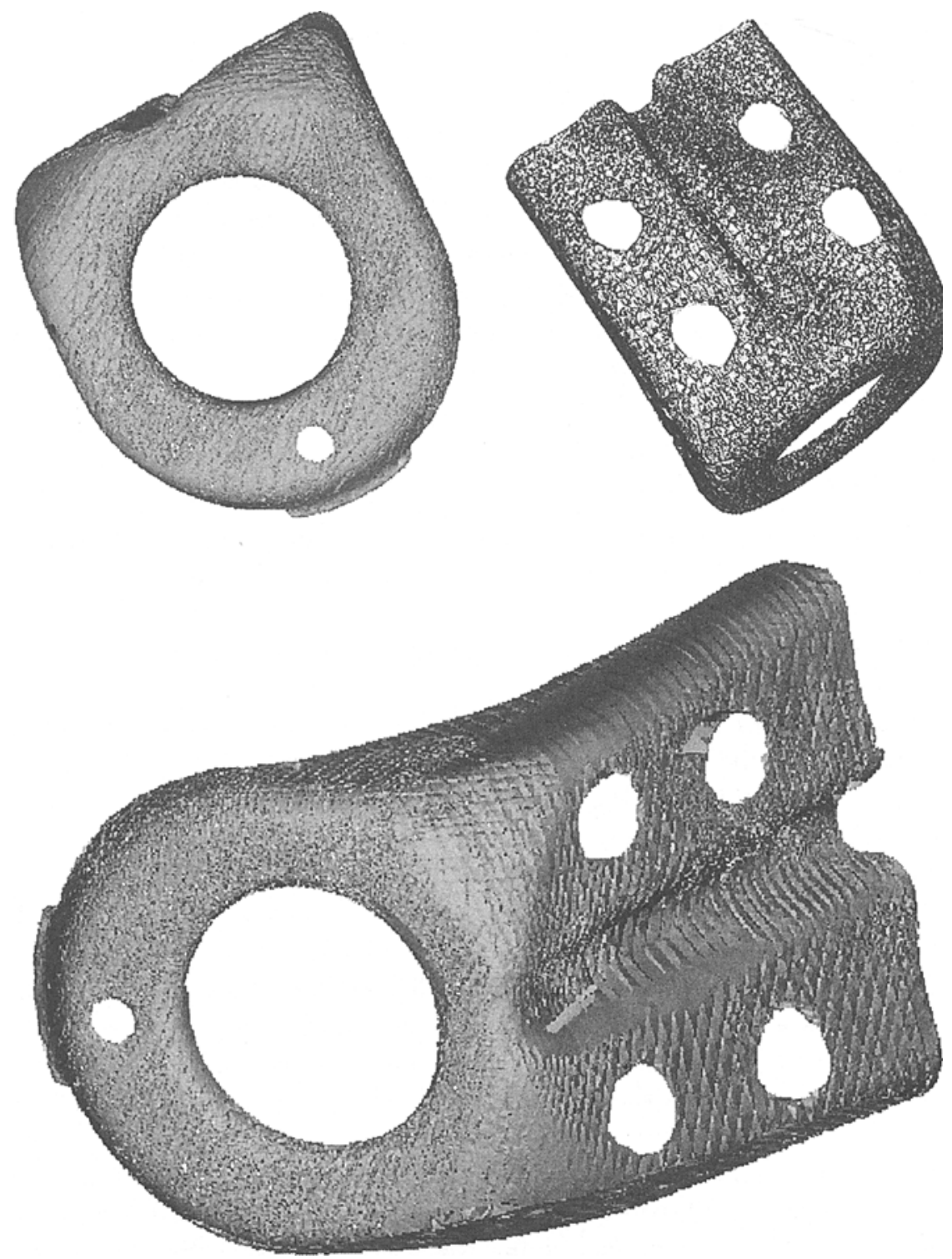

Fig. 23. The results by MOA approach for the same model as in Fig. 22. 
It can be seen that the final solid model obtained by the MOA algorithm together with the hole module is free from folded structures and holes. Other examples are shown from Figs. 14-21 and 23. In Figs. 14, 16 and 21, the most complex parts of the models are reconstructed effectively. In order to compare the results of MOA with other approaches, Hoppe's contour-tracing implicit function algorithm is selected. For the model with thin-slab structure, the results of both algorithms are shown in Figs. 22 and 23, respectively. In his approach, the holes are not reconstructed correctly, and redundant surfaces are generated in the region without any points and connected with the holes. His approach does not use the original points directly, which are used for determining one average approximate surface function. The nodes of the mesh are produced from this function. In this process, the points relating with geometric feature of the model also may be eliminated as the noise. Although the surface mesh has the good aspect ratio, it is not real one. On the other hand, in spite that MOA is not good at the treatment of the big noise, the shape of the model is reflected correctly.

\section{Conclusion}

In this study, a general algorithm called the "Maximum Opposite Angulation" is presented. The MOA algorithm, including the topology judgment is applicable to 3-D surface reconstruction problems as shown in the case studies.

The most important difference of this algorithm from the existing ones, appears in 3-D space as avoiding the projection plane completely used in [7], [22], and [14], which is considered to be essential, in constructing a complex 3-D surface, directly. The idea of finding the best internal angle configurations for the meshes in MOA algorithm differs considerably from the edge-swapping optimization of the existing algorithms in 3-D surface reconstructions.

The basis of the new method presented in this paper introduces a very general approach to 3-D surface reconstruction as discussed. The sorting of data points by forming line-segments from the shortest to the longest, introduces the base for the uniformity, which is essential for high quality meshes. This idea can actually be considered as a pre-optimization yielding the required quality network. The holes, which may appear on the surfaces of solid models, as discussed in detail, are removed from the model by "the loop module" which is indeed a topology judgment process.

The method is subject to further improvement for both solid meshes and for topology optimization. Another important improvement is related with the reduction of initial data set by identifying the scattered data points harming the homogeneity and the smoothness of the mesh architecture. In this improvement, "the list" is a good indicator for the evaluation of data by analyzing the initial distribution with statistical methods [11]. The authors are developing algorithms for such improvements as well.

It is possible to conclude that the MOA algorithm in 3-D mesh generation is a promising technique in precise surface reconstruction. The algorithm is comparable 
with the existing algorithms in terms of the quality of the final mesh architecture and the preservation of the geometrical features of the original surface.

Acknowledgement. The authors wish to gratefully thank to the anonymous referees for their corrections and remarks. And the research was supported by Japan Science and Technology Agency, Preventure Program. The patent was also submitted by their support (No. 2004-104732).

\section{References}

[1] N. Amenta, M. Bern and M. Kamvysselis, A new Voronoi-based surface reconstruction algorithm. Proceedings of SIGGRAPH '98, ACM, 1998, 415-421.

[2] C. Bajaj and G. Xu, Modeling scattered function data on curved surfaces. Fundamentals of Computer Graphics (eds. J. Chen et al.), World Scientific Publishing Co., 1994, 19-29.

[3] F. Bernardini, J. Mittleman, H. Rushmeier, C. Silva, and G. Taubin, The ball-pivoting algorithm for surface reconstruction. IEEE Trans. Visualization and Computer Graphics, $\mathbf{5}$, No. 4 (1999), 349-359.

[4] J. Boissonnat, Geometric structures for three dimensional shape representation. ACM Trans. Graphics, 3, No. 4 (1984), 266-286.

[5] A. Bowyer, Computing Dirichlet tessellations. The Computer Journal, 24, No. 2 (1981), 162-166.

[6] J.L. Brown, Vertex based data dependent triangulations. Computer Aided Geometric Design, 8, No. 3 (1991), 239-251.

[7] B.-K. Choi, H.-Y. Shin, Y.-I. Yoon and J.-W. Lee, Triangulation of scattered data in 3D space. Computer-Aided Design, 20, No. 5 (1988), 239-248.

[8] A.K. Cline and R.L. Renka, Storage efficient method for construction of a Thiessen triangulation. Rockey Mountain Journal of Mathematics, 14, No. 1 (1984), 119-139.

[9] R.A. Dwyer, A faster divide-and-conquer algorithm for constructing Delaunay triangulations. Algorithmica, 2, No. 2 (1987) 137-151.

[10] H. Edelsbrunner and E. Mücke, 3D alpha shapes. ACM Trans. Graphics, 13, No. 1 (1994), 43-72.

[11] O. Egorova, A.S. Gonenc and I. Hagiwara, Automatic mesh enhancement by control of aspect ratio distribution. Proceedings of the 15 th Computational Mechanics Conference of JSME, Kagoshima, 2002, 731-732.

[12] P.J. Green and R. Sibson, Computing Dirichlet tessellations in the plane. The Computer Journal, 21, No. 2 (1978), 168-173.

[13] B. Guo, J. Menon, and B. Willette, Surface reconstruction using alpha shapes. Computer Graphics Forum, 16, No.4 (1997), 177-190.

[14] H. Hoppe, Surface reconstruction from unorganized points. Doctoral Thesis, University of Washington, 1994.

[15] J. Huang and C.-H. Menq, Combinatorial manifold mesh reconstruction and optimization from unorganized points with arbitrary topology. Computer-Aided Design, 34, No. 2 (2002), 149-165.

[16] J. Katajainen and M. Koppinen, Constructing Delaunay triangulations by merging buckets in quad-tree order. Annales Sociatatis Mathematicae Polonae, Series IV, Fundamenta Infomaticae, 11, No. 3 (1988), 275-288.

[17] C.L. Lawson, Generation of a triangular grid with application to contour plotting. Jet Propulsion Laboratory Internal Technical Memorandum, No. 299, 1972.

[18] C.L. Lawson, Software for C1 Surface Interpolation, Mathematical Software 3 (ed. J.R. Rice). Academic Press, New York, 1977, 161-194.

[19] D.T. Lee and B.J. Schachter, Two algorithms for constructing a Delaunay Triangulation. International Journal of Computer and Information Sciences, 9, No. 3 (1980), 219-242.

[20] G.M. Nielson, A method for interpolating scattered data based upon minimum norm network. Mathematics of Computation, 40, No. 161 (1983), 253-271. 
[21] G.M. Nielson, Coordinate free scattered data interpolation. Topics in Multivariate Approximation (eds. L. Schumaker et al.), Academic Press, 1987, 175-184.

[22] D. Shepard, A two dimensional interpolation function for irregularly spaced data. Proceedings of the ACM National Conference, ACM Press, 1968, 517-524.

[23] R. Sibson, Locally equiangular triangulations. The Computer Journal, 21, No. 3 (1978), 243-245.

[24] K. Sugihara, Topology-oriented approach to robust geometric computation. Algorithms and Computation (ISSAC '99, Chennai, eds. A. Aggarwal and C.P. Rangan), Lecture Notes in Computer Science 1741, Springer, 1999, 357-366.

[25] W. Sun, C. Bradly, Y.-F. Zhang and H.-T. Loh, Cloud data modelling employing a unified non-redundant triangular mesh. Computer-Aided Design, 33 (2001), 183-193. 